\title{
NEUTRON-RICH NUCLEI POPULATED IN MULTI-NUCLEON TRANSFER REACTIONS: THE ${ }^{197} \mathrm{Au}+{ }^{130} \mathrm{Te}$ SYSTEM*
}

F. Galtarossa ${ }^{\mathrm{a}, \mathrm{b}}$, L. Corradi $^{\mathrm{a}}$, E. Fioretto $^{\mathrm{a}}$, D. Montanari $^{\mathrm{a}, \mathrm{c}}$ S. Szilner ${ }^{d}$, T. Mijatovićd ${ }^{d}$, G. Pollarolo ${ }^{e}$, D. ACKermann $^{f}$

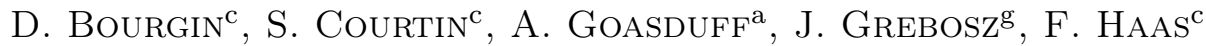
H.M. Jia ${ }^{\text {h }}$, D. JelaviĆ-Malenica ${ }^{\mathrm{d}}$, S. JeOnG ${ }^{\mathrm{i}}$, P.R. JohN ${ }^{\mathrm{j}}$ M. Milin ${ }^{d}$, G. Montagnoli ${ }^{\mathrm{j}}$, F. Scarlassara ${ }^{\mathrm{j}}$, N. SkUkan ${ }^{\mathrm{d}}$

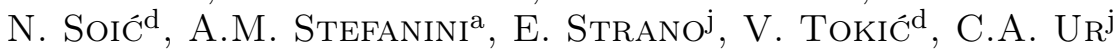
J.J. VALIEnte-Dobón ${ }^{\mathrm{a}}$, Y. WATANABE ${ }^{\mathrm{i}}$

a Istituto Nazionale di Fisica Nucleare, Laboratori Nazionali di Legnaro Legnaro, Italy

${ }^{\text {b}}$ Dipartimento di Fisica e Scienze della Terra, Università di Ferrara, Ferrara, Italy

${ }^{\mathrm{c}}$ Institut Pluridisciplinaire Hubert Curien, CNRS-IN2P3

Université de Strasbourg, Strasbourg, France

${ }^{\mathrm{d}}$ Ruđer Bošković Institute and University of Zagreb, Zagreb, Croatia

e Dipartimento di Fisica Teorica, Università di Torino

and Istituto Nazionale di Fisica Nucleare, Torino, Italy

${ }^{\mathrm{f}}$ GSI Helmholtzzentrum für Schwerionenforschung GmbH, Darmstadt, Germany

gThe H. Niewodniczański Institute of Nuclear Physics, PAN, Kraków, Poland

${ }^{\mathrm{h}}$ China Institute of Atomic Energy, Bejing, China

${ }^{i}$ Institute of Particle and Nuclear Studies, High Energy Accelerator Research

Organization (KEK), Tsukuba, Ibaraki, Japan

jDipartimento di Fisica, Università di Padova

and Istituto Nazionale di Fisica Nucleare, Padova, Italy

(Received January 17, 2017)

An experiment was performed at LNL to obtain information concerning the yield distributions in both the ${ }^{132} \mathrm{Sn}(N=82)$ and the ${ }^{208} \mathrm{~Pb}(N=126)$ regions of the nuclide chart. The experimental set-up was composed of the large-acceptance magnetic spectrometer PRISMA to detect the light partner of the reaction, in conjunction with a recently installed ancillary set-up to detect the heavy partner.

DOI:10.5506/APhysPolB.48.609

* Presented at the Zakopane Conference on Nuclear Physics "Extremes of the Nuclear Landscape", Zakopane, Poland, August 28-September 4, 2016. 


\section{Introduction}

Multi-nucleon transfer processes between heavy ions at energies close to the Coulomb barrier have been indicated as a competitive tool to produce and explore neutron-rich nuclei around the $N=126$ region of the nuclide chart [1,2], but very few experimental data exist so far. This region can be hardly accessed by fragmentation or fission reactions; moreover, the population of such heavy nuclei is considerably affected by secondary processes, such as particle evaporation and transfer-induced fission, which have the effect to shift the final yields to lower masses [3]. To understand how one can try to approach the neutron-rich heavy region, we have to keep in mind that transfer processes are governed by form factors and optimum $Q$-value considerations. With neutron-poor projectiles on heavy targets, only proton stripping and neutron pick-up channels are available, while with neutronrich projectiles also proton pick-up and neutron stripping channels open up. This corresponds, for the heavy partner, to the population in the "southeast" direction, leading to the neutron-rich heavy region.

The availability of beams with $A \sim 200$ at LNL made it possible to investigate these phenomena in more detail, making use of inverse kinematics to obtain a high kinetic energy of the recoils and hence the best possible ion identification in mass $A$ and nuclear charge $Z$. Following the experience gained in measurements in inverse kinematics at sub-barrier energy $[4,5]$, we performed an experiment to study the transfer channels in the reaction ${ }^{197} \mathrm{Au}+{ }^{130} \mathrm{Te}$ at $E_{\text {lab }}=1070 \mathrm{MeV}$. Concerning the "light" reaction product, the main goal was to determine the $A, Z$ and $Q$-value distributions and compare them with the predictions of theoretical models that have already proved to be successful with lower-mass systems [6-8]. In particular, ${ }^{130} \mathrm{Te}$ has just 2 protons more and 4 neutrons less than ${ }^{132} \mathrm{Sn}$, which represents a benchmark neutron-rich nucleus for a large number of physical phenomena. On the other hand, through the comparison between the yields of the heavy partner and those of the light, we can get quantitative information on the contribution of secondary processes.

\section{The experiment and the analysis}

We performed an experiment with a $2 \mathrm{pnA}{ }^{197} \mathrm{Au}$ beam delivered by the PIAVE-ALPI accelerator complex at LNL, at an energy of about $5.5 \mathrm{MeV} / A$, impinging on a $200 \mu \mathrm{g} / \mathrm{cm}^{2}{ }^{130} \mathrm{Te}$ target with a purity of $99.6 \%$.

The detection system (see Fig. 1) was composed of the large acceptance magnetic spectrometer PRISMA [9-11], placed close to the grazing angle, in kinematic coincidence with a new set-up acting as the second arm. This device consists of a Parallel Plate Avalanche Counter (PPAC), for timing and position information, followed by a Bragg chamber (BC) [12]. The BC 
operates according to the principles of Bragg Curve Spectroscopy (BCS) [13]. The Bragg peak (related to the $Z$ of the ions) and the total energy of the ions stopped into the active volume of the chamber are extracted by integrating the signals with shaping times of 0.25 and $4 \mu \mathrm{s}$, respectively.

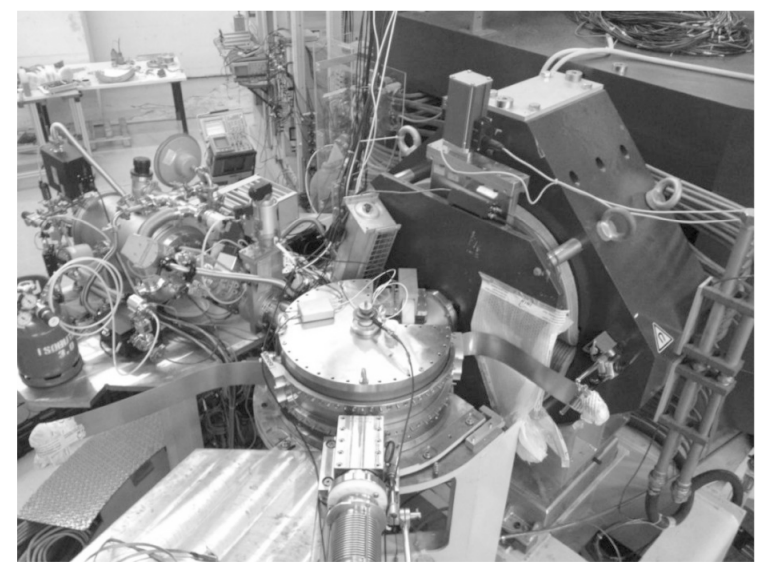

Fig. 1. A picture of the new sliding seal scattering chamber (in the center) with PRISMA on the right-hand side and the second arm on the left-hand side.

The coincident detection of both scattered ions in the present reaction required the two detectors to be placed at the same angle $\theta_{\text {lab }}= \pm 37^{\circ}$ with respect to the beam direction. The settings of PRISMA and BC were optimized for the detection of the light and heavy partners, respectively.

The nuclear charge of the ions detected in PRISMA is extracted through the $E-\Delta E$ method in the ionization chamber. Mass identification is then based on an event-by-event reconstruction of the ion trajectory, using also the position and timing information given by the entrance Micro-Channel Plate (MCP) detector and the Multi-Wire Parallel Plate Avalanche Counter (MWPPAC) focal plane detector.

Figure 2 shows the identification of light and heavy reaction products in the $E-\Delta E$ and $A / q$ versus focal plane position matrices. In the left panel, we can notice the overwhelming elastic channel at this bombarding energy for this heavy system. In this work, we will focus on neutron-transfer channels, reconstructing the masses of Te isotopes $(Z=52)$. In this procedure, one has to be aware that mass discrimination of the outgoing reaction products detected in PRISMA becomes more and more critical with very heavy ions. Therefore, a proper analysis has to be followed to separate the different isotopes at a level sufficient to be able to derive absolute yields. In particular, the mass resolution is affected by the presence of optical aberrations, especially for non-central trajectories, which have to be properly taken into 

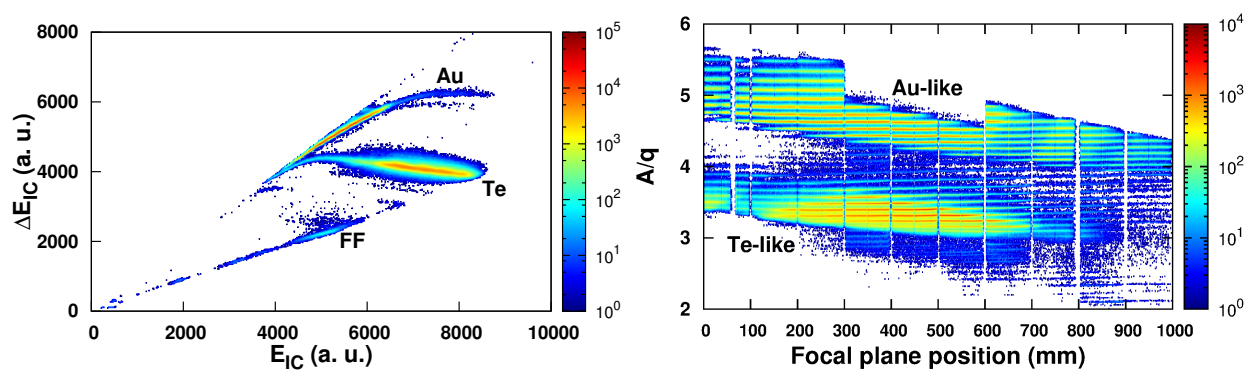

Fig. 2. $E-\Delta E$ matrix for the present experiment (left), where Te-like, Au-like and fission fragments (FF) detected in PRISMA are labelled and $A / q$ versus focal plane position (right) where light and heavy partners of the reaction can be clearly distinguished.

account in the ion trajectory reconstruction. For this reason, a proper algorithm has been developed [14]: a least-square polynomial fit of the $A / q$ lines for a given charge state versus entrance $x$ and $y$ position on the MCP and exit position on the focal plane has been performed iteratively and a further fine correction of the "wavy" structures in the connection points between different sections of the MWPPAC (see left panel of Fig. 3) is applied. The result of this procedure is illustrated in Fig. 3. The final mass resolution obtained is $\Delta A / A \sim 1 / 250$. One can see that most of the neutron-transfer flux is along the stripping direction (here stripping is referred to the Te-like ions).
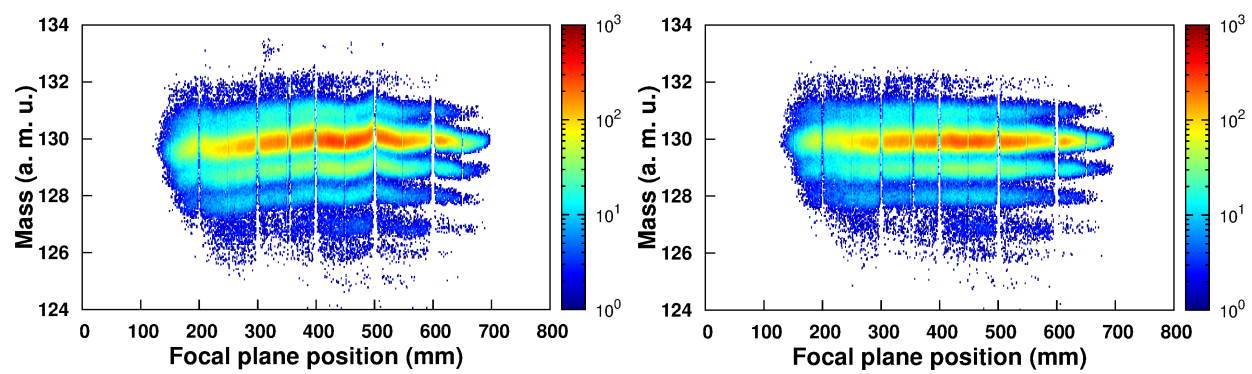

Fig. 3. Matrices of Te mass $(Z=52)$ versus focal plane position before (left) and after (right) the empirical corrections described in the text.

The PRISMA data analysis has been implemented including information coming from the second arm. In the kinematic coincidence, PRISMA always acts as a master trigger for the data acquisition system. When set in coincidence with the second arm, we determined the mass of the binary partners by measuring the time-of-flight difference between PRISMA and the PPAC and the correlated angles of both fragments. As said before, the Bragg peak 
amplitude versus energy provides $Z$ discrimination. As an example, Fig. 4 shows a clear separation between light and heavy reaction fragments in the $\mathrm{BC}$, whose pressure was optimized for the heavy ones.

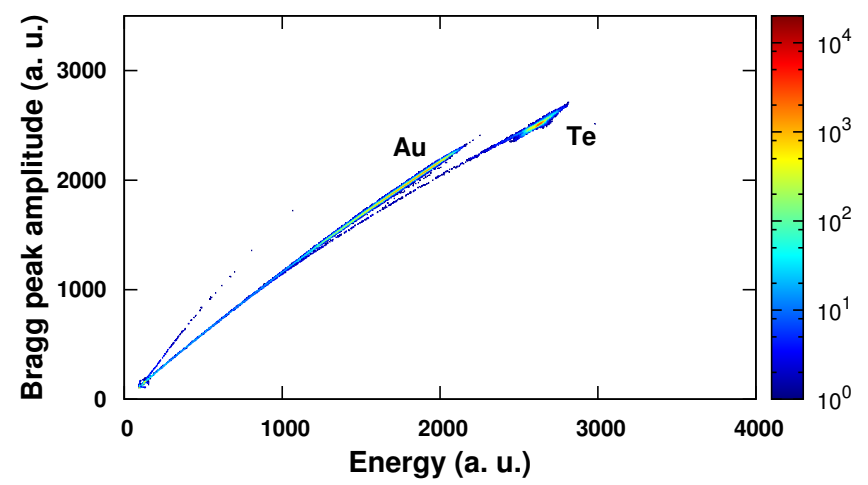

Fig. 4. Matrix of the Bragg peak amplitude versus the total energy released in the BC. Clearly distinguished Te-like and Au-like ions are labelled.

The mass of the ions detected in the second arm was determined through a kinematic formula derived in the hypothesis of pure binary reaction. Figure 5 shows the obtained mass distribution where both reaction partners are visible. Resolution for the heavy partner is $\sim 4.5 \mathrm{u}(\sim 2.3 \%) \mathrm{FWHM}$ and is mainly affected by the short path length for the time of flight between the MCP of PRISMA and the PPAC of the second arm.

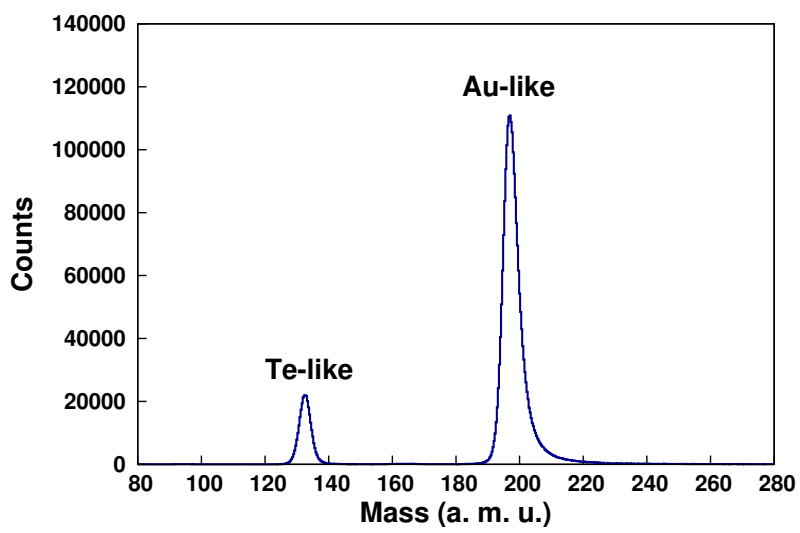

Fig. 5. Reconstructed binary partner mass in the second arm where light and heavy reaction products are clearly separated. 
In the forthcoming analysis, we will compare the yields of Te isotopes with theoretical models to get quantitative information on neutron-transfer channels and the effect of evaporation for the light partner of the reaction. Concerning the heavy partner, we aim at extracting quantitative information on secondary processes like transfer-induced fission and particle evaporation.

The authors are grateful to the LNL Tandem-ALPI staff for the good quality beams and the target laboratory for the excellent targets. This work was partially supported by the European Union Seventh Framework Program FP7/2007-2013 under grant agreement No. 262010 ENSAR and in part by the Croatian Science Foundation under the project 7194 .

\section{REFERENCES}

[1] C.H. Dasso, G. Pollarolo, A. Winther, Phys. Rev. Lett. 73, 1907 (1994).

[2] Y.X. Watanabe et al., Phys. Rev. Lett. 115, 172503 (2015).

[3] L. Corradi, G. Pollarolo, S. Szilner, J. Phys. G: Nucl. Part. Phys. 36, 113101 (2009).

[4] D. Montanari et al., Phys. Rev. Lett. 113, 052501 (2014).

[5] L. Corradi et al., Phys. Rev. C 84, 034603 (2011).

[6] A. Winther, Nucl. Phys. A 572, 191 (1994).

[7] A. Winther, Nucl. Phys. A 594, 203 (1995).

[8] A. Winther, GRAZING, http:/www.to.infn.it/ nanni/grazing

[9] A.M. Stefanini et al., Nucl. Phys. A 701, 217c (2002).

[10] S. Szilner et al., Phys. Rev. C 76, 024604 (2007).

[11] L. Corradi et al., Nucl. Instrum. Methods Phys. Res. B 317, 743 (2013).

[12] E. Fioretto, J. Phys.: Conf. Ser. 533, 012006 (2014).

[13] C.R. Gruhn et al., Nucl. Instrum. Methods Phys. Res. 196, 33 (1982).

[14] A. Vogt, Phys. Rev. C 92, 024619 (2015). 